\title{
Combination treatment with 2-methoxyestradiol overcomes bortezomib resistance of multiple myeloma cells
}

In-Sung Song, Yu Jeong Jeong, Seung Hun Jeong, Hye Jin Heo, Hyoung Kyu Kim, Sung Ryul Lee, Tae Hee Ko, Jae Boum Youm, Nari Kim, Kyung Soo Ko, Byoung Doo Rhee and Jin Han

Bortezomib is a proteasome inhibitor used for the treatment of relapsed/refractory multiple myeloma (MM). However, intrinsic and acquired resistance to bortezomib has already been observed in MM patients. In a previous report, we demonstrated that changes in the expression of mitochondrial genes lead to changes in mitochondrial activity and bortezomib susceptibility or resistance, and their combined effects contribute to the differential sensitivity or resistance of MM cells to bortezomib. Here we report that the combination treatment of bortezomib and 2-methoxyestradiol (2ME), a natural estrogen metabolite, induces mitochondria-mediated apoptotic cell death of bortezomib-resistant MM KMS20 cells via mitochondrial reactive oxygen species (ROS) overproduction. Bortezomib plus 2ME treatment induces a higher level of cell death compared with treatment with bortezomib alone and increases mitochondrial ROS and $\mathrm{Ca}^{2}+$ levels in KMS20 cells. Pretreatment with the antioxidant $\mathrm{N}$-acetyl-L-cysteine scavenges mitochondrial ROS and decreases cell death after treatment with bortezomib plus $2 \mathrm{ME}$ in KMS20 cells. Moreover, we observed that treatment with bortezomib plus $2 \mathrm{ME}$ maintains the activation of $c$-Jun $\mathrm{N}$-terminal kinase (JNK) and mitogen-activated protein kinase kinase kinase 4/7 (MKK4/7). Collectively, combination treatment with bortezomib and $2 \mathrm{ME}$ induces cell death via JNK-MKK4/7 activation by overproduction of mitochondrial ROS. Therefore, combination therapy with specific mitochondrial-targeting drugs may prove useful to the development of novel strategies for the treatment of bortezomib-resistant MM patients.

Experimental \& Molecular Medicine (2013) 45, e50; doi:10.1038/emm.2013.104; published online 25 October 2013

Keywords: bortezomib; combination therapy; multiple myeloma; 2-methoxyestradiol; resistance; superoxide dismutase 2

\section{INTRODUCTION}

Multiple myeloma (MM) is a neoplastic plasma cell disorder characterized by clonal proliferation of malignant plasma cells in the bone marrow. ${ }^{1}$ Strategies for MM treatment have focused on the development of anticancer drugs that reduce the number of abnormal plasma cells in affected patients. Bortezomib is a boronate-based, dipeptidyl proteasome inhibitor that has been approved for use by the United States Food and Drug Administration to treat relapsed/ refractory $\mathrm{MM}$ that primarily targets the chymotrypsin-like activity of the intracellular proteasome enzyme complex. ${ }^{2,3}$ However, bortezomib therapy can cause both intrinsic and acquired resistance in MM patients. ${ }^{4,5}$ In numerous cases, targeted compounds have shown limited efficacy when used as monotherapies, likely due to the multi-step process of tumorigenesis. ${ }^{6,7}$ Therefore, there is a growing need for the development of optimal combinations of these novel agents to improve the survival of patients suffering from myeloma. Recently, we reported a role for mitochondria in resistance to bortezomib-induced cell death and found that human MM KMS20 cells were resistant to bortezomib due to potentiation of mitochondrial function. ${ }^{8}$ In that report, changes in the expression levels of certain genes, such as cyclophilin D, the mitochondrial calcium uniporter, and superoxide dismutase 2 , led to changes in both mitochondrial activity and bortezomib susceptibility or resistance; their combined effects contributed to differential sensitivity or resistance of MM cells to bortezomib. Specifically, we showed that changes in mitochondrial reactive oxygen species (ROS) levels via depletion of superoxide dismutase 2 and treatment

National Research Laboratory for Mitochondrial Signaling, Department of Physiology, College of Medicine, Cardiovascular and Metabolic Disease Center, Inje University, Busan, Korea

Correspondence: Professor J Han, National Research Laboratory for Mitochondrial Signaling, Department of Physiology, College of Medicine, Cardiovascular and Metabolic Disease Center, Inje University, 633-165 Gaegeum-dong, Busanjin-gu, Busan 614-735, Korea.

E-mail: phyhanj@inje.ac.kr

Received 25 April 2013; revised 23 July 2013; accepted 1 August 2013 
with 2-methoxyestradiol (2ME), known as a SOD inhibitor, induced bortezomib-resistant MM cell death. However, the mechanisms that regulate the enhancement of cell death after treatment of bortezomib-resistant KMS20 cells with a combination of $2 \mathrm{ME}$ and bortezomib remain unknown.

The compound $2 \mathrm{ME}$ is a natural metabolite of the endogenous estrogens 17b-estradiol and 2-hydroxyestradiol. ${ }^{9}$ Recent studies showed that $2 \mathrm{ME}$ exerts both anti-tumor and anti-angiogenic activities on various tumors, and $2 \mathrm{ME}$ is currently in a clinical trial as a cancer treatment. ${ }^{10}$ The antitumor mechanism of $2 \mathrm{ME}$ is multi-faceted, including the induction of G1 or G2/M cell cycle arrest and tumor cell apoptosis; the increased expression of FAS, p53 and p21WAF1; the activation of $c$-jun $N$-terminal activated kinase (JNK); the inhibition of tubulin polymerization; the formation of ROS; the mitochondrial release of cytochrome $c .^{11-19}$ In recent years, many clinical trials have evaluated the therapeutic efficacy of $2 \mathrm{ME}$ against MM. However, little is known about the molecular events implicated in the ability of $2 \mathrm{ME}$ to overcome bortezomib resistance.

In this study, we investigated the anti-myeloma activity of the combination treatment of $2 \mathrm{ME}$ and bortezomib in bortezomib-resistant KMS20 cells. We found that this combined treatment induces mitochondria-mediated cell death via mitochondrial ROS overproduction and JNK activation in bortezomib-resistant KMS20 cells. Moreover, we present evidence that the initial event in the mechanism of action of $2 \mathrm{ME}$ is the induction of ROS in mitochondria, which leads to bortezomib resistance.

\section{MATERIALS AND METHODS}

\section{Reagents}

Bortezomib was purchased from Selleck Biochemicals (Houston, TX, USA). The fluorescent dyes tetramethylrhodamine, ethyl ester, perchlorate (TMRE), and rhod-2AM were purchased from Invitrogen (Carlsbad, CA, USA). The chemicals $2 \mathrm{ME}, \mathrm{N}$-acetyl-L-cysteine (NAc), and L-buthionine-sulfoximine were purchased from Sigma-Aldrich (St Louis, MO, USA).

\section{Cell culture}

KMS20, KMS26 and KMS28BM cells were originally obtained from the Kawasaki Medical School (Kurashiki, Japan) and were maintained in RPMI 1640 (Lonza). The culture medium was supplemented with $10 \%$ fetal bovine serum (Lonza) and $1 \%$ antibiotic-antimycotic solution (Invitrogen), and cells were grown in a $5 \% \mathrm{CO}_{2}$ incubator.

\section{Western blot and subcellular fractionation}

Cells were lysed in lysis buffer A (20 mM HEPES (pH 7.5), $1 \mathrm{~mm}$ EDTA, 2 mм EGTA, $150 \mathrm{~mm} \mathrm{NaCl,} \mathrm{10 \%} \mathrm{glycerol,} \mathrm{1 \%} \mathrm{Triton} \mathrm{X-100}$ and protease cocktail I/II (Sigma-Aldrich)). Proteins were separated by sodium dodecyl sulfate-polyacrylamide gel electrophoresis and transferred onto nitrocellulose membranes; membranes were blocked with $5 \%$ skim milk in $0.01 \mathrm{~m}$ Tris-buffered saline ( $\mathrm{pH} 7.5$ ) containing $0.5 \%$ Tween 20 and incubated with the appropriate primary antibodies. For cytochrome $c$ release after bortezomib plus $2 \mathrm{ME}$ treatment, subcellular fractions were obtained using the ProteoExtract Subcellular Proteome Extraction Kit (Calbiochem, La Jolla, CA, USA). The purity of each fraction was verified using the following selective markers: $\alpha$-tubulin (cytosolic marker) and mitochondrial protein peroxiredoxin III (mitochondrial marker). ${ }^{20}$

\section{Flow cytometry for detection of cell death}

To estimate cell death, fluorescein isothiocyanate-conjugated, annexin $\mathrm{V}$-specific antibody was labeled with propidium iodide (PI), according to the manufacturer's instructions (BD Biosciences, Franklin Lakes, NJ, USA). Annexin V- and/or PI-positive cells were analyzed by FACSCanto flow cytometry (BD Biosciences). Cell cycle distribution was determined by DNA staining with PI (Sigma). A total of $1 \times 10^{6}$ cells were collected and fixed in $70 \%$ ethanol. Cell pellets were suspended in PI and simultaneously treated with RNase at $37^{\circ} \mathrm{C}$ for $30 \mathrm{~min}$. The percentages of cells in different phases of the cell cycle were measured using a FACSCalibur flow cytometer (BD Biosciences).

\section{Measurement of mitochondrial ROS}

Mitochondrial ROS generation was assessed using Mito-Sox red (Molecular Probes, Eugene, OR, USA). KMS20 cells were seeded onto $30-\mathrm{mm}$ culture dishes at a density of $3 \times 10^{5}$ cells and incubated with $1 \mu \mathrm{M}$ Mito-Sox for $20 \mathrm{~min}$ at $37^{\circ} \mathrm{C}$. For quantitative analysis of ROS generation, Mito-Sox-treated cells were analyzed by flow cytometry using a FACSCantoII instrument. Fluorescence images of Mito-Sox-loaded cells were acquired using a confocal laser scanning microscope (LSM700, Carl-Zeiss, Oberkochen, Germany) and analyzed using Axiovision microscope software, version 4.8.2 (Carl-Zeiss).

\section{Mitochondria membrane potential analysis}

Mitochondrial membrane potential $\left(\Delta \Psi_{\mathrm{m}}\right)$ was assessed in KMS20 cells using the $\Delta \Psi_{\mathrm{m}}$-specific fluorescent dye, TMRE. Cells $\left(1 \times 10^{6}\right)$ from each group were incubated with $200 \mathrm{~nm}$ TMRE for $20 \mathrm{~min}$ at $37^{\circ} \mathrm{C}$. TMRE-loaded cells were analyzed using a FACSCantoII flow cytometer (BD Biosciences). Fluorescence images of TMRE-loaded cells were acquired using a confocal laser scanning microscope (Carl-Zeiss).

\section{Mitochondria calcium concentration assay}

Mitochondria $\mathrm{Ca}^{2+}$ relative concentrations were analyzed in KMS20 cells using the mitochondrial $\mathrm{Ca}^{2+}$-sensitive fluorescent dye, rhod2AM. A cold/warm incubation protocol was used to exclusively load mitochondria with rhod-2AM. Briefly, cells $\left(1 \times 10^{6}\right.$ cells per sample $)$ were washed with phosphate-buffered saline and stained with $5 \mu \mathrm{M}$ rhod-2AM in normal Tyrode's solution containing $143 \mathrm{~mm} \mathrm{NaCl}$, $5.4 \mathrm{~mm} \mathrm{KCl}, 1.8 \mathrm{~mm} \mathrm{CaCl}_{2}, 0.5 \mathrm{~mm} \mathrm{MgCl}_{2}, 5.5 \mathrm{~mm}$ glucose and $5 \mathrm{~mm}$ HEPES (pH 7.4 with $\mathrm{KOH}$ ) for $120 \mathrm{~min}$ at $4{ }^{\circ} \mathrm{C}$, followed by a 30 -min incubation at $37^{\circ} \mathrm{C}$. Rhod-2AM-loaded cells were analyzed using a FACSCantoII flow cytometer (BD Biosciences). Fluorescence images of TMRE-loaded cells were acquired using a confocal laser scanning microscope (Carl-Zeiss).

\section{Statistical analysis}

Data were analyzed using the Student's $t$-test and SigmaPlot 8.0 software; $P$ values were derived to assess statistical significance as follows: ${ }^{\star} P<0.05 ;{ }^{*} P<0.01$; and ${ }^{*}{ }^{*} P<0.001$. Data for all figures are expressed as the means \pm standard deviation (s.d.) of three independent experiments. 


\section{RESULTS}

Combination treatment with bortezomib plus $2 \mathrm{ME}$ induces cell death of bortezomib-resistant KMS20 cells

We previously showed that mitochondrial functional differences, including $\Delta \Psi_{\mathrm{m}}$, oxygen consumption rate, ATP production and $\mathrm{Ca}^{2}+$ retention capacity, were involved in the susceptibility of MM cells to undergo bortezomib-mediated cell death. ${ }^{8}$ In that report, we showed that MM cell lines had different susceptibilities against bortezomib according to their mitochondrial function. Specifically, KMS20 cells were resistant to bortezomib-mediated cell death. As shown in Figure 1a, fewer than $30 \%$ of KMS20 cells underwent cell death, even after $75 \mathrm{~nm}$ bortezomib treatment. We have now extended our work to investigate the combination of bortezomib and 2ME in bortezomib-resistant KMS20 cells.

We first determined the single effects of $2 \mathrm{ME}$ in bortezomibresistant KMS20 cells and bortezomib-sensitive KMS28BM cells before examining the combination effects of $2 \mathrm{ME}$. As shown in Figure 1b, cell death was induced slightly in bortezomib-resistant KMS20 cells but was induced in over $50 \%$ of bortezomib-sensitive KMS28BM cells. Therefore, KMS20 cells showed resistance after a single treatment with either bortezomib or 2ME. To examine the beneficial effect of combination treatment with bortezomib and 2ME, we performed flow cytometric analyses after labeling with PI/annexin V-fluorescein isothiocyanate in KMS20 cells. KMS20 cells were cultured in different concentrations of bortezomib containing $1 \mu \mathrm{M} 2 \mathrm{ME}$ for $48 \mathrm{~h}$. Combination treatment significantly increased cell death in KMS20 and KMS26 cells in a bortezomib dose-dependent manner (Figure 1c). To verify whether the effects of combination treatment were induced by apoptosis, sub-G1 phase DNA content was measured after bortezomib or combination treatment of KMS20 cells. DNA content analysis also revealed a population of cells in the sub-G1 hypodiploid phase, indicating that apoptosis was significantly increased in KMS20 cells treated with bortezomib plus $2 \mathrm{ME}$ in a bortezomib dose-dependent manner (Figure 1d).

Immunoreactive bands for cleaved caspase- 8 and caspase- 3 were more intense in lysates after combined treatment than in lysates after a single treatment (Figure 2a). We examined whether mitochondria were involved in combined treatmentinduced cell death. In the mitochondrial apoptotic pathway, the signaling mediator between the cytosol and mitochondria is cytochrome $c$. Thus, cytochrome $c$ release was compared in bortezomib-treated KMS20 cells and in cells treated with both bortezomib and 2ME. Cytochrome $c$ release was markedly increased in the cytosol of cells treated with both compounds compared with cells treated only with bortezomib (Figure 2b). As a result of this release, caspase- 3 activation was subsequently enhanced in cells receiving the combination treatment compared with cells receiving a single treatment (Figure 2a). These results indicate that KMS20 is a bortezomib-resistant MM cell line and that combination treatment with $2 \mathrm{ME}$ can induce a cell death mechanism in these bortezomib-resistant cells. Moreover, we reported that mitochondrial activity contributes to the differential sensitivity or resistance of MM cells to bortezomib in our previous study. Thereby, we propose that the combination treatment of $2 \mathrm{ME}$ and bortezomib may induce cell death via the regulation of mitochondria activity of bortezomib-resistant KMS20 cells.

Combination treatment with bortezomib and $2 \mathrm{ME}$ induces mitochondrial dysfunction via overproduction of mitochondrial ROS in KMS20 cells

To investigate the connection between induction of cell death by combination treatment and mitochondrial activity in bortezomib-resistant MM, we examined mitochondrial a

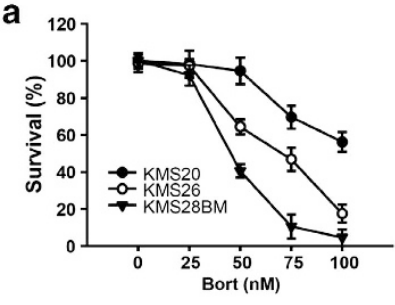

C

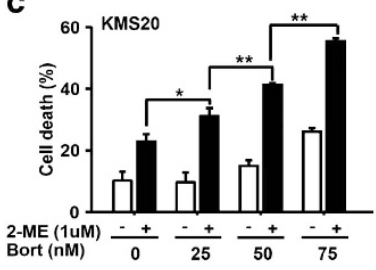

b
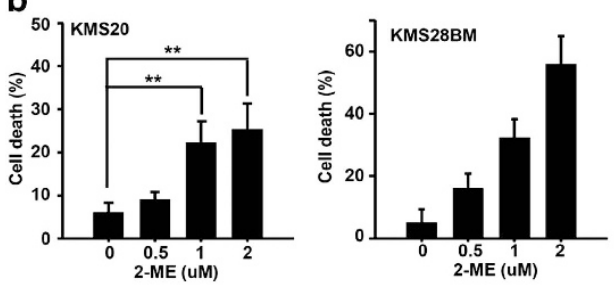

d Vehicle Bort (75nM)
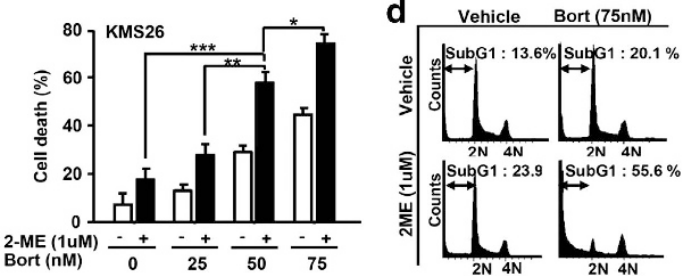

Figure 1 Combination effects of 2-methoxyestradiol (2ME) and bortezomib on apoptosis of KMS20 cells. (a) KMS cells were incubated with different doses of bortezomib, and cell viability was determined by fluorescence-activated cell sorting (FACS) analysis after propidium iodide (PI)/annexin V-based staining. (b) KMS20 (left panel) and KMS28BM (right panel) cells were treated with 2ME at the indicated doses for $48 \mathrm{~h}$ and subjected to FACS analysis as described above. (c) KMS20 (left panel) and KMS26 (right panel) cells were treated with bortezomib plus $2 \mathrm{ME}$ for $48 \mathrm{~h}$ at the indicated doses, and cell death was measured as described above. (d) Sub-G1 percentage (apoptotic cell population) was measured by Pl staining and flow cytometry after $48 \mathrm{~h}$. 
a
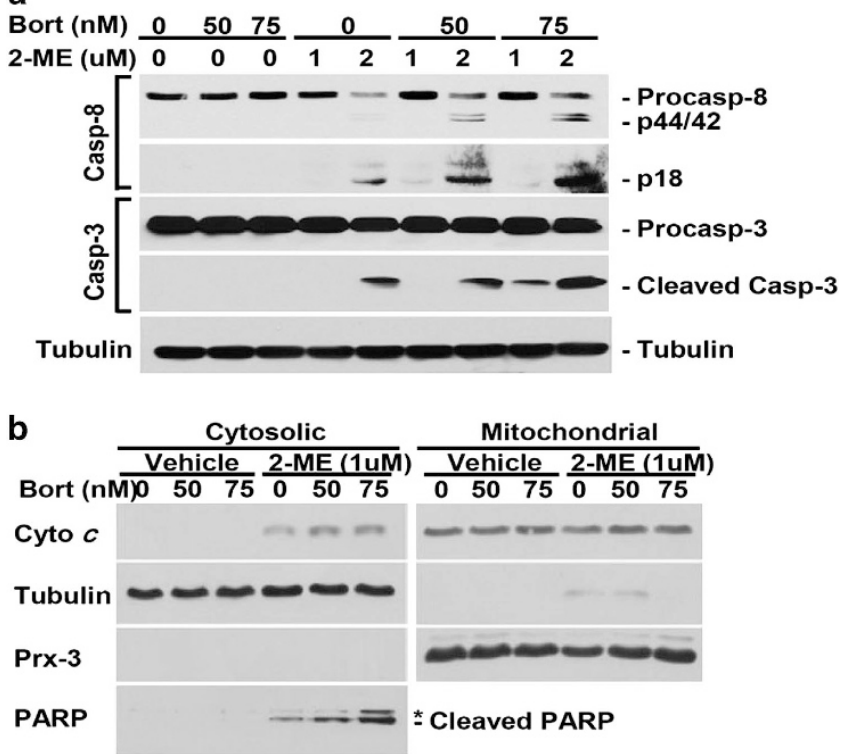

Figure 2 Combination treatment with bortezomib plus 2-methoxyestradiol (2ME) induces caspase activation via a mitochondriamediated intrinsic apoptotic pathway. (a) KMS20 cells were treated with bortezomib plus $2 \mathrm{ME}$ at the indicated doses for $48 \mathrm{~h}$ and subjected to western blotting using the indicated antibodies. (b) KMS20 cells were treated with bortezomib plus $2 \mathrm{ME}$ for $48 \mathrm{~h}$, and cells were separated into cytosolic and mitochondrial fractions. Tubulin and Prx3 were used as cytosolic and mitochondrial markers, respectively. PARP, poly (ADP-ribose) polymerase. function by assessing $\left[\mathrm{Ca}^{2+}\right]_{\mathrm{M}}, \Delta \Psi_{\mathrm{m}}$ and mitochondrial ROS production. First, we measured mitochondrial ROS levels using an oxidant-sensitive fluorescent dye, Mito-SOX red. While bortezomib treatment alone did not trigger superoxide anion production in KMS20 cells, the combination treatment resulted in a remarkable increase in mitochondrial ROS levels (Figures $3 \mathrm{a}$ and d). Next, we determined whether the increase in mitochondrial $\mathrm{Ca}^{2+}$ concentrations contributes to cytotoxicity after combination treatment. KMS20 cells were incubated in the presence of different bortezomib concentrations plus $2 \mathrm{ME}(1 \mu \mathrm{M})$ for $48 \mathrm{~h}$, treated with rhod-2AM and subjected to flow cytometry (Figure $3 \mathrm{~b}$ ). We observed an increase in $\left[\mathrm{Ca}^{2+}\right]_{\mathrm{M}}$ after bortezomib plus $2 \mathrm{ME}$ treatment. However, the increase in $\left[\mathrm{Ca}^{2+}\right]_{M}$ was independent of the dose of bortezomib, as shown in Figure 3b. We next examined the effects of bortezomib plus $2 \mathrm{ME}$ on the levels of $\Delta \Psi_{\mathrm{m}}$ in KMS20 cells. The levels of $\Delta \Psi_{\mathrm{m}}$ were measured using TMRE, which accumulates in mitochondria via a $\Delta \Psi_{\mathrm{m}}$-driven process. Because uptake of TMRE into mitochondria depends on $\Delta \Psi_{\mathrm{m}}$, a collapse of $\Delta \Psi_{\mathrm{m}}$ is associated with a decrease in the fluorescence intensity of TMRE. Flow cytometric analyses of red fluorescence indicated that the combination treatment of KMS20 cells exhibited a depolarization of $\Delta \Psi_{\mathrm{m}}$ and that this response increased according to the dosage of bortezomib as the level of mitochondrial ROS (Figure 3c). Taken together, these data suggest that increased mitochondrial ROS levels may cause combination treatment-induced cell death in KMS20 cells.
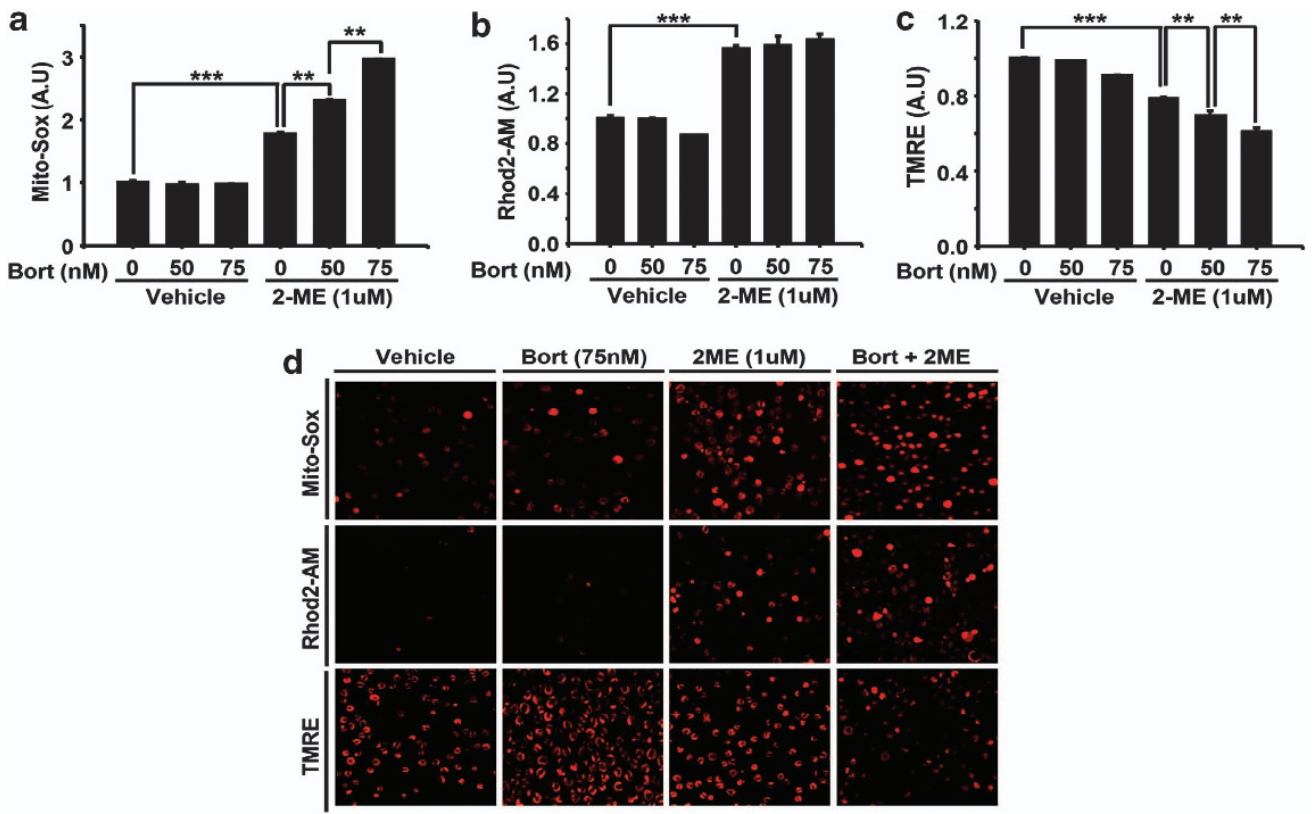

Figure 3 Combination treatment with bortezomib plus 2-methoxyestradiol (2ME) enhances mitochondrial reactive oxygen species production, leading to mitochondrial dysfunction. (a) KMS20 cells were treated with bortezomib plus $2 \mathrm{ME}$ at the indicated doses, subjected to flow cytometry after staining with Mito-Sox and quantified. (b) Mitochondrial $\mathrm{Ca}^{2}+$ concentrations in KMS20 cells were measured before and after treatment with bortezomib and/or 2ME using a FACSCantoll instrument. (c) Mitochondrial membrane potential $\left(\Delta \Psi_{m}\right)$ in KMS20 cells treated with bortezomib plus $2 \mathrm{ME}$ was measured by tetramethylrhodamine, ethyl ester, perchlorate (TMRE) staining and fluorescence-activated cell sorting analysis. (d) KMS20 cells were treated with bortezomib plus $2 \mathrm{ME}$ for $48 \mathrm{~h}$, and cells were stained with Mito-Sox, rhod-2AM and TMRE, and analyzed by confocal microscopy. 
Increased mitochondrial ROS levels after bortezomib plus 2ME treatment induces KMS20 cell death via prolonged JNK activation

To determine whether the increase in mitochondrial ROS levels causes cell death in response to combination treatment, we used NAc as an antioxidant. ${ }^{16}$ We found that NAc successfully scavenged mitochondrial ROS after treatment with bortezomib plus 2ME in KMS20 cells (Figure 4a). Next, we examined the effects of treatment with bortezomib plus $2 \mathrm{ME}$ and NAc in KMS20 cells by performing a western blot (Figure 4b). KMS20 cells treated with bortezomib plus $2 \mathrm{ME}$ were more sensitive to bortezomib-mediated cell death than a single treatment with bortezomib. Pretreatment with NAc reduced the rate of cell death that had increased after combination treatment with 2ME and bortezomib in KMS20 cells. Moreover, the effects of combination treatment were fully reversed by the addition of zVAD-fmk, whereas it was not increased by the addition of L-buthionine-sulfoximine, a known pro-oxidant. These results suggest that the increase of ROS via $2 \mathrm{ME}$ treatment can overcome bortezomib resistance. Therefore, we performed flow cytometric analyses after labeling with PI/annexin V-fluorescein isothiocyanate in KMS20 cells to confirm these results (Figure 4c). As shown in Figure $4 c$, the pattern of cell death was identical to the western blotting results. Especially, increase of cell death by addition of L-buthionine-sulfoximine was not observed as well as in western blot. These results indicate that cell death induced by treatment with $2 \mathrm{ME}$ plus bortezomib likely involves mitochondrial ROS overproduction. Additionally, JNK, whose activation is sustained by mitochondrial ROS, ${ }^{21}$ was also phosphorylated after combination treatment. From these data, we propose that activation of JNK is actively involved in the induction of cell death after bortezomib plus $2 \mathrm{ME}$ treatment.

To determine the regulators of apoptotic cell death after treatment with bortezomib plus $2 \mathrm{ME}$, we analyzed the activity of mitogen-activated protein kinase (MAPK) associated with cell death induced by mitochondrial ROS in KMS20 cells. Stress-activated JNK and p38 regulate a variety of intracellular processes in response to environmental stresses, cytokines, ROS and growth factors. KMS20 cells treated with either bortezomib alone or bortezomib plus $2 \mathrm{ME}$ for $48 \mathrm{~h}$ were subjected to western blot analysis using anti-p-p38MAPK and p-SAPK/JNK antibodies (Figure 5a). Phosphorylated
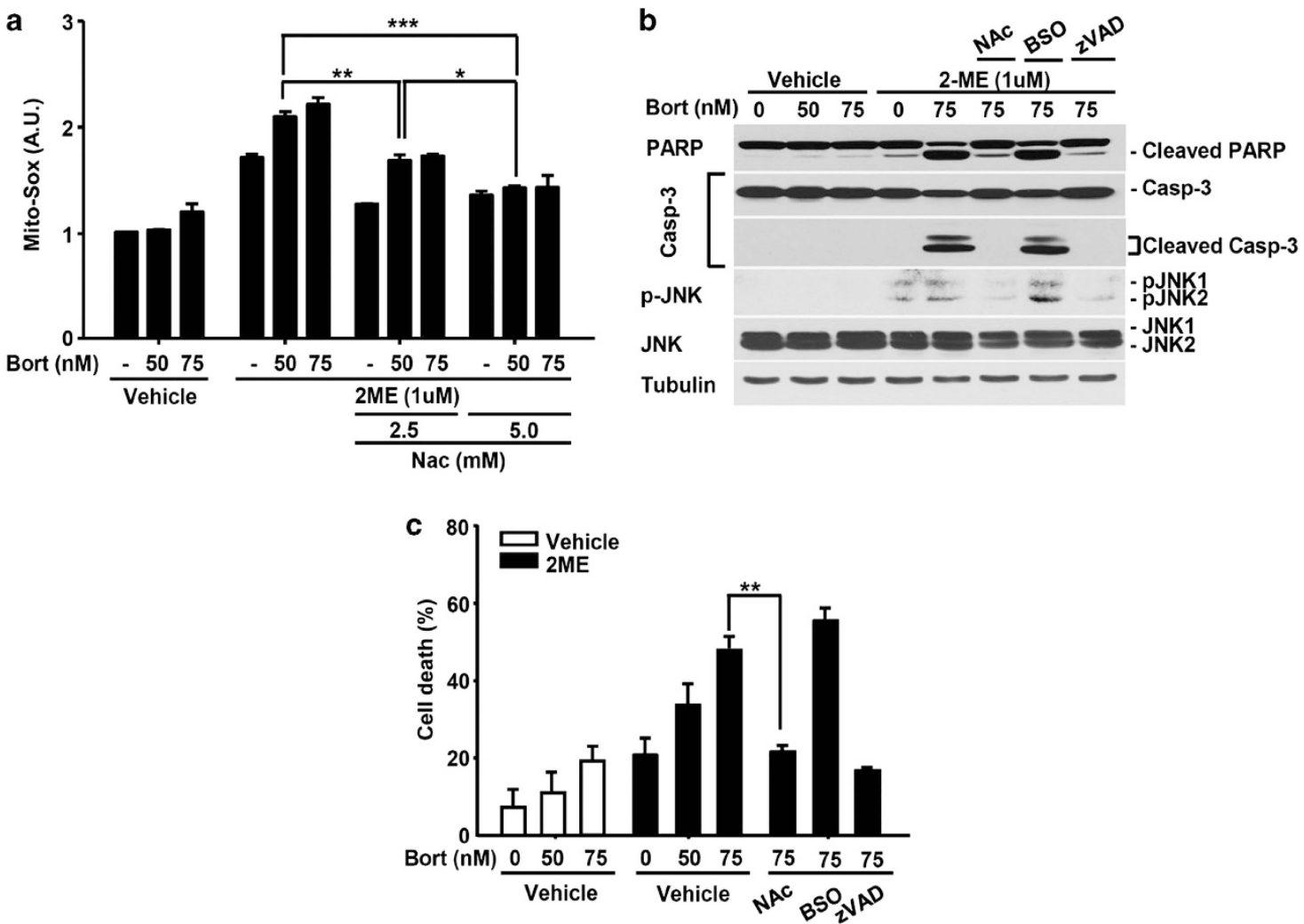

Figure 4 Suppression of bortezomib/2-methoxyestradiol (2ME)-induced mitochondrial reactive oxygen species (ROS) accumulation in $\mathrm{N}$-acetyl-L-cysteine (NAc)-treated KMS20 cells. (a) KMS20 cells were pretreated with the indicated doses of NAc for $1 \mathrm{~h}$, and co-treated with bortezomib plus $2 \mathrm{ME}$ for $48 \mathrm{~h}$. Mitochondrial ROS production was measured using a FACSCantoll instrument after Mito-Sox staining. (b, c) KMS20 cells were pretreated with NAc ( $5 \mathrm{~mm})$, L-buthionine-sulfoximine (BSO) and zVAD-fmk ( $10 \mu \mathrm{m})$ for $1 \mathrm{~h}$ as indicated on the figure, and co-treated with bortezomib plus $2 \mathrm{ME}$ for $48 \mathrm{~h}$. (b) Cells were subjected to western blotting using the indicated antibodies and (c) cell viability was determined by fluorescence-activated cell sorting analysis after propidium iodide/annexin V-based staining. PARP, poly (ADP-ribose) polymerase. 
a

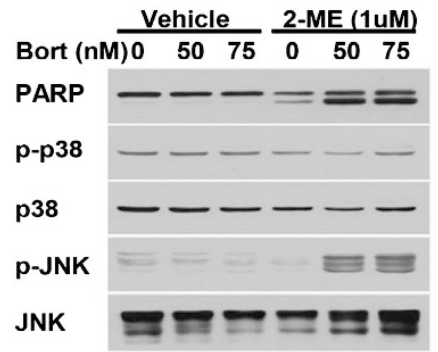

b

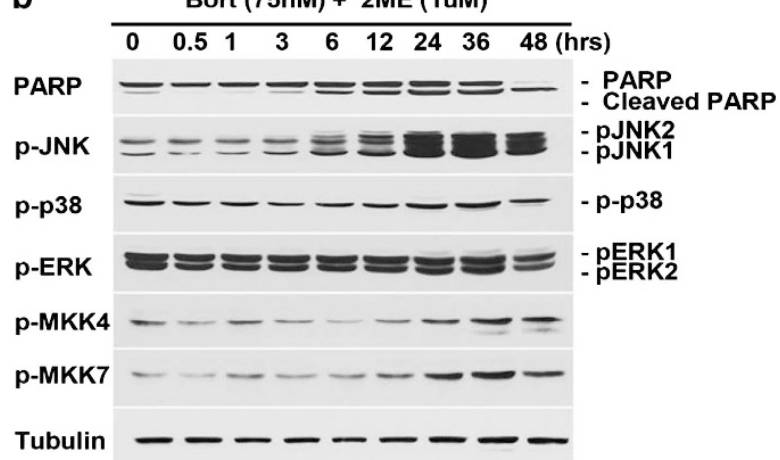

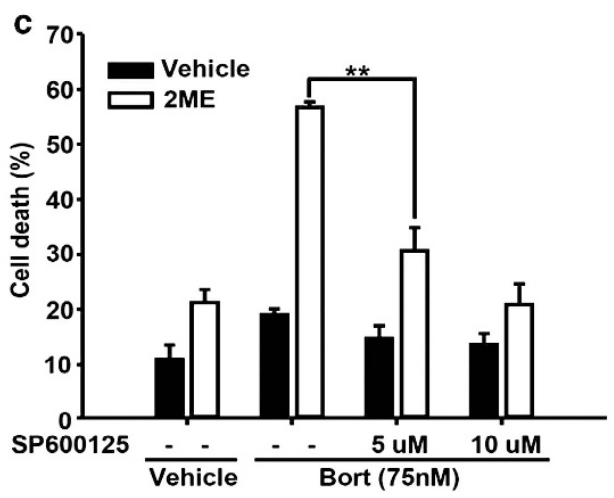

Figure 5 Involvement of c-Jun $N$-terminal kinase (JNK) phosphorylation, but not p38 and ERK, in the acceleration of cell death induced by bortezomib plus 2-methoxyestradiol (2ME). (a) Cells were treated with bortezomib plus $2 \mathrm{ME}$ for $48 \mathrm{~h}$ and subjected to western blotting analysis with the indicated antibodies. The same membrane was reprobed with antibodies to JNK, p38MAPK or ERK. (b) The effects of combination treatment on the sustained activation of JNK in KMS20 cells are shown after exposure to bortezomib plus $2 \mathrm{ME}$ for the indicated times. The cells were subjected to western blotting analysis with the indicated antibodies. (c) Cells were treated with bortezomib and $2 \mathrm{ME}$ in the presence or absence of $10 \mu \mathrm{moll}-1$ of SP600125 (JNK-specific inhibitor). Cell death percentage representing annexin $\mathrm{V}(+)$ /propidium iodide $(+)$-stained cells, which were analyzed by flow cytometry. PARP, poly (ADP-ribose) polymerase.

p38MAPK levels were unchanged, even after combined treatment, whereas phosphorylation of JNK increased after bortezomib plus $2 \mathrm{ME}$ treatment.

To further investigate the mechanism of cell death after combination treatment with bortezomib and 2ME, KMS20 cells were treated with bortezomib plus $2 \mathrm{ME}$ at various times. Treatment with both bortezomib and 2ME sustained JNK activation at later time points, whereas, as expected from previous data, neither p38MAPK nor ERK activation was detected in KMS20 cells. Moreover, JNK is known to be activated by both MKK4 and MKK7.22 Figure 5b shows a sustained phase of MKK4/MKK7 phosphorylation, in accordance with the cleavage of poly (ADP-ribose) polymerase, after combination treatment with bortezomib and $2 \mathrm{ME}$. Additionally, it is noteworthy that cell death in the Bort/ 2ME-treated cells was dramatically reduced when a SP600125, a JNK inhibitor, was co-treated (Figure $5 \mathrm{c}$ ). Together, these data demonstrate that combination treatment with bortezomib and 2ME prolongs MKK4/7-JNK activation by an overproduction of ROS due to mitochondrial dysfunction and that a sustained MKK4/7-JNK activation has a crucial role in cell death.

\section{DISCUSSION}

Bortezomib is a dipeptidyl boronic acid derivative that inhibits the chymotrypsin-like activity of proteasomes; it has received United States Food and Drug Administration approval for the treatment of relapsed/refractory MM. However, a majority of patients with relapsed or refractory MM do not respond to such treatment, and resistance is common. ${ }^{23,24}$ Here, we show that the treatment of bortezomib-resistant MM cells with bortezomib plus $2 \mathrm{ME}$ induces cell death via mitochondrial dysfunction, which regulates resistance to apoptosis in response to bortezomib. Thus, to develop an effective therapeutic regimen for bortezomib-resistant $M M$, we evaluated the effects of this combination treatment on mitochondria dysfunction so as to increase cell death susceptibility in response to bortezomib. We demonstrated different susceptibilities of three MM cell lines: KMS20, KMS26 and KMS28BM (Figure 1). KMS20 cells were shown to be quite resistant to bortezomib treatment. Even the highest concentration (100 nm bortezomib) failed to induce more than $50 \%$ apoptosis in KMS20 cells. On the other hand, KMS20 cells treated with bortezomib plus 2ME displayed abrupt apoptosis in a bortezomib dose-dependent manner (Figures 


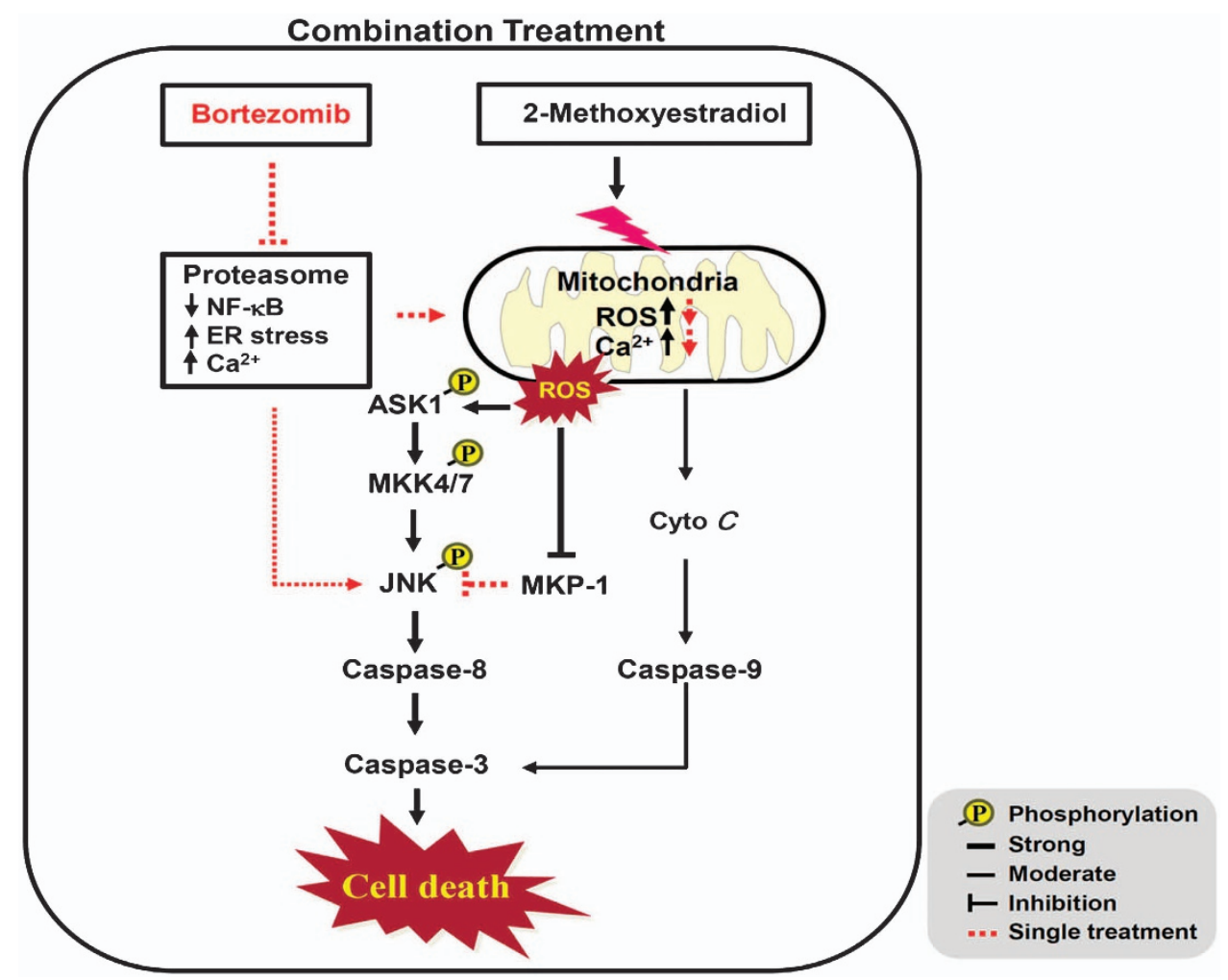

Figure 6 Proposed mechanism for the acceleration of cell death induced by bortezomib plus 2-methoxyestradiol (2ME) treatment of bortezomib-resistant multiple myeloma (MM) cells. The cytotoxic effect of bortezomib seems to arise partially from inhibition of the antiapoptotic transcription factor nuclear factor B. Bortezomib has also effects on MM biology, such as dysregulating $\mathrm{Ca}^{2+}$ homeostasis and endoplasmic reticulum (ER) stress-induced apoptosis (red line). However, single-bortezomib therapy can cause both intrinsic and acquired resistance in MM patients. In bortezomib-resistant MM cells, bortezomib alone failed to reach a critical threshold to induce apoptosis because of improvements in mitochondrial activities, such as reactive oxygen species (ROS) elimination via increases in antioxidant proteins, membrane potential and calcium capacity, resulting in the negative regulation of the mitochondrial-mediated apoptotic cell death pathway (red lines). Conversely, when bortezomib plus 2ME treatment is used, overproduction of ROS in mitochondria is induced, diffusing ROS outside of mitochondria and blocking both mitogen-activated protein kinase phosphatases and MKK4/7. This causes the activation of c-Jun $\mathrm{N}$-terminal kinase (JNK), via phosphorylation and activation downstream of ASK1, which is activated via ROS, eventually leading to the activation of a caspase cascade and cell death (black lines).

1c and d). Moreover, when bortezomib and 2ME were administered in a dose-dependent manner, procaspase- 8 was cleaved into activated caspase- 8 (Figure 2). Cleaved caspases- 8 activate procaspase- 3 , which induces cells to undergo apoptosis. Cytochrome $c$ is also released from mitochondria by activated caspase- 8 . From these data, we believe that the combination treatment of bortezomib plus $2 \mathrm{ME}$ is a novel, promising therapy for bortezomib-resistant MM.

In a previous report, we showed that changes in mitochondrial activity contribute to differential sensitivity or resistance of MM cells to bortezomib. ${ }^{8}$ Therefore, we examined the sensitizing effects of combination treatment on mitochondrial function (Figure 3). Mitochondrial ROS levels, after combination treatment with bortezomib and $2 \mathrm{ME}$, were dramatically increased and dependent on bortezomib dose (Figure 3a). Mitochondrial $\mathrm{Ca}^{2}+$ levels increased regardless of bortezomib dose after combination treatment (Figure 3b), whereas $\Delta \Psi_{\mathrm{m}}$ drastically declined according to bortezomib dose (Figure $3 \mathrm{c}$ ). These data suggest that increased mitochondrial ROS levels regulate cell death after combination treatment with bortezomib and $2 \mathrm{ME}$.

To evaluate the role of mitochondrial ROS, KMS20 cells were treated with bortezomib plus $2 \mathrm{ME}$ in the presence or absence of inhibitors for caspase (zVAD-fmk) and ROS (NAc). As shown in Figure 4a, mitochondrial ROS levels that were increased after combination treatment were attenuated by pretreatment with NAc. Cell death induced by combination treatment was reduced by pretreatment with zVAD-fmk and NAc, suggesting that the effects of combination treatment on MM cell death involve coordination among caspases and ROSdependent pathways. This interpretation is based on the increase in apoptosis seen after $2 \mathrm{ME}$, presumably via a mitochondria redox-dependent mechanism. ${ }^{25}$ The hypothesis that ROS has a critical role in apoptotic signal transduction has emerged from several observations. ROS production induced by $2 \mathrm{ME}$ was previously described in hepatocellular carcinoma cells, ${ }^{15}$ and treatment of leukemia cells with $2 \mathrm{ME}$ resulted in apoptosis via the generation of superoxide anion. ${ }^{13}$ 
Moreover, induction of JNK activation by $\mathrm{H}_{2} \mathrm{O}_{2}$ was recently demonstrated in several cell lines. ${ }^{26-28}$ Generally, activation of JNK and p38 MAPK is known to be highly responsible to ROS shift, implicating its additional role as a ROS sensor of cells. Cell survival and death are tightly correlated with early/ transient and late/sustained activation of JNK and p38, respectively, under oxidative conditions. ${ }^{29-31}$ This implies that oxidative cell death is dependent on the extent and/or duration of MAPKs activation.

Our data show that JNK phosphorylation is linked to cell death after combination treatment with bortezomib plus $2 \mathrm{ME}$ (Figure 4b). JNK activation and cell death induction after combination treatment were both decreased by pretreatment with NAc and zVAD-fmk. In addition, $\mathrm{H}_{2} \mathrm{O}_{2}$ has been shown to activate all three types of MAPKs in diverse cell types. ${ }^{16,32-36}$ We have confirmed the activation of all three types of MAPKs in KMS20 cells after bortezomib and 2ME treatment (Figure 5a). JNK activation, but neither p38 nor ERK activation, was consistently induced after combination treatment. These data suggest that cell death after combination treatment with bortezomib plus $2 \mathrm{ME}$ depends on JNK activation via increased ROS levels due to mitochondrial dysfunction. However, the JNK activation pathway has both pro- and anti-apoptotic properties. ${ }^{37}$ In other words, sustained JNK activation is predominantly associated with induction of apoptosis, whereas transient JNK activation is involved in cell survival. ${ }^{38}$ As shown in Figure 5b, our results clearly show that the sustained activation of JNK, which may be attributable to the inactivation of MAPK phosphatases by ROS, ${ }^{21,39,40}$ was induced by bortezomib plus $2 \mathrm{ME}$ treatment in parallel with poly (ADP-ribose) polymerase cleavage. In contrast, the activation of both p38 and ERK was maintained after combination treatment of KMS20 cells. Furthermore, our data show a sustained phase of MKK4/7 phosphorylation (a known activator of JNK), in accordance with poly (ADPribose) polymerase cleavage, in KMS20 cells treated with bortezomib plus 2ME. These results suggest that mitochondrial ROS after combination treatment acts to regulate only JNK activation in KMS20 cells. Collectively, these findings allow us to propose a comprehensive model for the role of combination treatment with bortezomib and $2 \mathrm{ME}$ in bortezomib-resistant MM cells (Figure 6). Among the proteins known to be involved in redox signaling, ASK1 is the best-characterized ROS target molecule. ${ }^{41}$ ASK1 activation, induced by ROS, results in the activation of the MKK-JNK pathway. ${ }^{42,43}$ Moreover, mitochondrial ROS leads to the prolonged activation of JNK via the inactivation of MAPK phosphatases..$^{21,39,40}$ Eventually, prolonged activation of JNK leads to the activation of caspases and cell death.

In summary, this study reports for the first time that combination treatment with bortezomib and $2 \mathrm{ME}$ is a novel therapeutic strategy for bortezomib-resistant MM; combination treatment facilitates the leak of ROS due to mitochondrial dysfunction, sustains the activation of the JNK/caspase axis and induces bortezomib/2ME-mediated cell death in bortezomibresistant MM. Given that combination treatment specifically induces the death of bortezomib-resistant $\mathrm{MM}$ cells, the combination of bortezomib and 2ME offers excellent translational potential and promises to be a novel MM therapy.

\section{CONFLICT OF INTEREST}

The authors declare no conflict of interest.

\section{ACKNOWLEDGEMENTS}

This study was supported by a grant from the National R\&D Program for Cancer Control, Ministry for Health, Welfare and Family affairs, Republic of Korea (0920040), a Priority Research Centers Program through the National Research Foundation of Korea (NRF) funded by the Ministry of Education, Science and Technology (2010-0020224) and a Basic Science Research Program through the National Research Foundation of Korea(NRF) funded by the Ministry of Education, Science and Technology (2012R1A1A2041700).

1 Kyle RA, Rajkumar SV. Multiple myeloma. N Engl J Med 2004; 351: 1860-1873.

2 Rajkumar SV, Richardson PG, Hideshima T, Anderson KC. Proteasome inhibition as a novel therapeutic target in human cancer. J Clin Oncol 2005; 23: 630-639.

3 Chauhan D, Li G, Podar K, Hideshima T, Mitsiades C, Schlossman R et al. Targeting mitochondria to overcome conventional and bortezomib/proteasome inhibitor PS-341 resistance in multiple myeloma (MM) cells. Blood 2004; 104: 2458-2466.

4 Caldera H, Giralt S. Stem cell transplantation for multiple myeloma: current status and future directions. Curr Hematol Rep 2004; 3: 249-256.

5 Richardson PG, Barlogie B, Berenson J, Singhal S, Jagannath S, Irwin D et al. A phase 2 study of bortezomib in relapsed, refractory myeloma. N Eng/ J Med 2003; 348: 2609-2617.

6 Ocio EM, Mateos MV, Maiso P, Pandiella A, San-Miguel JF. New drugs in multiple myeloma: mechanisms of action and phase $1 / / \mathrm{l}$ clinical findings. Lancet Oncol 2008; 9: 1157-1165.

7 Hanahan D, Weinberg RA. The hallmarks of cancer. Cell 2000; 100 57-70.

8 Song IS, Kim HK, Lee SR, Jeong SH, Kim N, Ko KS et al. Mitochondrial modulation decreases the bortezomib-resistance in multiple myeloma cells. Int J Cancer 2013; 133: 1357-1367.

9 Seegers JC, Aveling ML, Van Aswegen CH, Cross M, Koch F, Joubert WS The cytotoxic effects of estradiol-17 beta, catecholestradiols and methoxyestradiols on dividing MCF-7 and HeLa cells. J Steroid Biochem 1989; 32: 797-809.

10 Sutherland TE, Anderson RL, Hughes RA, Altmann E, Schuliga M, Ziogas J et al. 2-Methoxyestradiol-a unique blend of activities generating a new class of anti-tumour/anti-inflammatory agents. Drug Discov Today 2007; 12: 577-584.

11 Attalla $H$, Westberg JA, Andersson LC, Adlercreutz H, Makela TP. 2-Methoxyestradiol-induced phosphorylation of $\mathrm{Bcl}-2$ : uncoupling from JNK/SAPK activation. Biochem Biophys Res Commun 1998; 247: 616-619.

12 Hamel E, Lin CM, Flynn E, D'Amato RJ. Interactions of 2-methoxyestradiol, an endogenous mammalian metabolite, with unpolymerized tubulin and with tubulin polymers. Biochemistry 1996; 35: 1304-1310.

13 Huang P, Feng L, Oldham EA, Keating MJ, Plunkett W. Superoxide dismutase as a target for the selective killing of cancer cells. Nature 2000; 407: 390-395.

14 Kumar AP, Garcia GE, Slaga TJ. 2-methoxyestradiol blocks cell-cycle progression at $\mathrm{G}(2) / \mathrm{M}$ phase and inhibits growth of human prostate cancer cells. Mol Carcinog 2001; 31: 111-124.

15 Lin HL, Liu TY, Chau GY, Lui WY, Chi CW. Comparison of 2-methoxyestradiol-induced, docetaxel-induced, and paclitaxel-induced apoptosis in hepatoma cells and its correlation with reactive oxygen species. Cancer 2000; 89: 983-994.

16 Song IS, Kim SU, Oh NS, Kim J, Yu DY, Huang SM et al. Peroxiredoxin contributes to TRAIL resistance through suppression of redox-sensitive 
caspase activation in human hepatoma cells. Carcinogenesis 2009; 30: 1106-1114.

17 Mukhopadhyay T, Roth JA. Induction of apoptosis in human lung cancer cells after wild-type p53 activation by methoxyestradiol. Oncogene 1997; 14: 379-384.

18 Qadan LR, Perez-Stable CM, Anderson C, D'Ippolito G, Herron A, Howard GA et al. 2-Methoxyestradiol induces G2/M arrest and apoptosis in prostate cancer. Biochem Biophys Res Commun 2001; 285: 1259-1266.

19 Qanungo S, Basu A, Das M, Haldar S. 2-Methoxyestradiol induces mitochondria dependent apoptotic signaling in pancreatic cancer cells. Oncogene 2002; 21: 4149-4157.

20 Song IS, Jun SY, Na HJ, Kim HT, Jung SY, Ha GH et al. Inhibition of MKK7-JNK by the TOR signaling pathway regulator-like protein contributes to resistance of HCC cells to TRAIL-induced apoptosis. Gastroenterology 2012; 143: 1341-1351.

21 Ventura JJ, Cogswell P, Flavell RA, Baldwin AS Jr., Davis RJ. JNK potentiates TNF-stimulated necrosis by increasing the production of cytotoxic reactive oxygen species. Genes Dev 2004; 18: 2905-2915.

22 Derijard B, Raingeaud J, Barrett T, Wu IH, Han J, Ulevitch RJ et al. Independent human MAP-kinase signal transduction pathways defined by MEK and MKK isoforms. Science 1995; 267: 682-685.

23 Richardson PG, Hideshima T, Mitsiades C, Anderson KC. The emerging role of novel therapies for the treatment of relapsed myeloma. J Natl Compr Canc Netw 2007; 5: 149-162.

24 Lonial S, Waller EK, Richardson PG, Jagannath S, Orlowski RZ, Giver CR et al. Risk factors and kinetics of thrombocytopenia associated with bortezomib for relapsed, refractory multiple myeloma. Blood 2005; 106: 3777-3784.

25 Djavaheri-Mergny M, Wietzerbin J, Besancon F. 2-Methoxyestradiol induces apoptosis in Ewing sarcoma cells through mitochondrial hydrogen peroxide production. Oncogene 2003; 22: 2558-2567.

26 Benhar M, Engelberg D, ROS Levitzki A. Stress-activated kinases and stress signaling in cancer. EMBO Rep 2002; 3: 420-425.

27 Aoki H, Kang PM, Hampe J, Yoshimura K, Noma T, Matsuzaki M et al. Direct activation of mitochondrial apoptosis machinery by c-Jun N-terminal kinase in adult cardiac myocytes. J Biol Chem 2002; 277: 10244-10250.

28 Hashimoto M, Hsu LJ, Rockenstein E, Takenouchi T, Mallory M, Masliah E. alpha-Synuclein protects against oxidative stress via inactivation of the c-Jun N-terminal kinase stress-signaling pathway in neuronal cells. J Biol Chem 2002; 277: 11465-11472.

29 Chen YR, Wang X, Templeton D, Davis RJ, Tan TH. The role of c-Jun $\mathrm{N}$-terminal kinase (JNK) in apoptosis induced by ultraviolet $\mathrm{C}$ and gamma radiation. Duration of JNK activation may determine cell death and proliferation. J Biol Chem 1996; 271: 31929-31936.

30 Guo YL, Baysal K, Kang B, Yang LJ, Williamson JR. Correlation between sustained $\mathrm{C}$-Jun $\mathrm{N}$-terminal protein kinase activation and apoptosis induced by tumor necrosis factor-alpha in rat mesangial cells. J Biol Chem 1998; 273: 4027-4034.
31 Roulston A, Reinhard C, Amiri P, Williams LT. Early activation of c-Jun N-terminal kinase and p38 kinase regulate cell survival in response to tumor necrosis factor alpha. J Biol Chem 1998; 273: 10232-10239.

32 Martindale JL, Holbrook NJ. Cellular response to oxidative stress: signaling for suicide and survival. J Cell Physiol 2002; 192: 1-15.

33 Sano M, Fukuda K, Sato T, Kawaguchi H, Suematsu M, Matsuda S et al. ERK and p38 MAPK, but not NF-kappaB, are critically involved in reactive oxygen species-mediated induction of IL- 6 by angiotensin II in cardiac fibroblasts. Circ Res 2001; 89: 661-669.

34 Song IS, Kim HK, Jeong SH, Lee SR, Kim N, Rhee BD et al. Mitochondrial peroxiredoxin III is a potential target for cancer therapy. Int $\mathrm{J} \mathrm{Mol} \mathrm{Sci}$ 2011; 12: 7163-7185.

35 Guyton KZ, Liu Y, Gorospe M, Xu Q, Holbrook NJ. Activation of mitogenactivated protein kinase by $\mathrm{H}_{2} \mathrm{O}_{2}$. Role in cell survival following oxidant injury. J Biol Chem 1996; 271: 4138-4142.

36 Chen K, Vita JA, Berk BC, Keaney JF Jr. C-Jun N-terminal kinase activation by hydrogen peroxide in endothelial cells involves SRC-dependent epidermal growth factor receptor transactivation. J Biol Chem 2001; 276: 16045-16050.

37 Davis RJ. Signal transduction by the JNK group of MAP kinases. Cell 2000; 103: 239-252.

38 Ventura JJ, Hubner A, Zhang C, Flavell RA, Shokat KM, Davis RJ. Chemical genetic analysis of the time course of signal transduction by JNK. Mol Cell 2006; 21: 701-710.

39 Papa S, Zazzeroni F, Bubici C, Jayawardena S, Alvarez K, Matsuda S et al. Gadd45 beta mediates the NF-kappa B suppression of JNK signalling by targeting MKK7/JNKK2. Nat Cell Biol 2004; 6: 146-153.

40 Sakon S, Xue X, Takekawa M, Sasazuki T, Okazaki T, Kojima Y et al. NF-kappaB inhibits TNF-induced accumulation of ROS that mediate prolonged MAPK activation and necrotic cell death. EMBO J 2003; 22: 3898-3909.

41 Kang SW, Chang TS, Lee TH, Kim ES, Yu DY, Rhee SG. Cytosolic peroxiredoxin attenuates the activation of Jnk and p38 but potentiates that of Erk in Hela cells stimulated with tumor necrosis factor-alpha. J Biol Chem 2004; 279: 2535-2543.

42 Gotoh Y, Cooper JA. Reactive oxygen species- and dimerizationinduced activation of apoptosis signal-regulating kinase 1 in tumor necrosis factor-alpha signal transduction. J Biol Chem 1998; 273: 17477-17482.

43 Saitoh M, Nishitoh H, Fujii M, Takeda K, Tobiume K, Sawada Y et al. Mammalian thioredoxin is a direct inhibitor of apoptosis signal-regulating kinase (ASK) 1. EMBO J 1998; 17: 2596-2606.

(c) (i) (2) This work is licensed under a Creative Commons Attribution-NonCommercial-ShareAlike 3.0 Unported License. To view a copy of this license, visit http:// creativecommons.org/licenses/by-nc-sa/3.0/ 